\title{
Primer Congreso Colombiano de Obstetricia y Ginecología
}

\author{
(Recuento histórico) \\ Fernando Sánchez Torres*
}

La ginecobstetricia es hoy en Colombia una de las especialidades médicas de mayor avanzada, como que es, así mismo, una de las más antiguas. Vale recordar que durante la segunda mitad del siglo XIX, cuando apenas comenzaba la medicina nacional a despuntar, ya había médicos dedicados al oficio de los partos. Basta mencionar los nombres de Leoncio Barreto, José María Buendía, Rafael Rocha Castilla, Librado Rivas y Joaquín Maldonado, para aceptar que fue así. Por su parte, la disciplina ginecológica se confundía con la cirugía, pues era esencialmente quirúrgica. No obstante, hubo también en aquellas calendas médicos "especialistas en enfermedades de la mujer", como el cubano Salvador Riera y la doctora Ana Galvis Hotz, graduada en Suiza. Pero fue en 1903, en el Hospital San Juan de Dios de Bogotá, cuando el profesor Rafael Ucrós Durán fundó la primera cátedra de ginecología.

Durante la primera mitad del siglo actual ambas especialidades fueron tomando mayor auge, influidas las dos por las corrientes francesas. De ahí que se mantuvieran divorciadas hasta la década de los sesenta. Como el Primer Congreso Colombiano de Obstetricia y Ginecología tuvo ocurrencia en 1953, conviene echar una mirada a la situación que rodeaba entonces el transcurrir de esas dos especialidades.

\section{Estado de la Obstetricia y la Ginecología en Colombia, promediando el siglo $\mathrm{XX}$}

Al iniciar el decenio de los cuarenta, Colombia contaba con cuatro facultades de medicina, todas de carácter oficial. En ellas se enseñaban por aparte la obstetricia y la ginecología, y los profesores eran obstetras o ginecólogos puros. En la misma década se fundó la primera escuela de medicina privada, adscrita a la Pontificia Universidad Javeriana, que siguió el mismo esquema docente, puesto que los catedráticos habían sido formados en la Universidad Nacional, a su vez heredera de la tradición francesa.

Aun cuando todavía se atendían partos a domicilio, existía la tendencia, sobre todo en las clases sociales pudientes económicamente, a que los nacimientos ocurrieran en instituciones asistenciales privadas. Las mujeres de pobre condición socio-económica acudían a los hospitales públicos o de caridad. Esta circunstancia permitía que las maternidades oficiales fueran un rico filón para la docencia, un campo de práctica excepcional. En Bogotá, que fue la ciudad donde se reunió el Congreso que motiva este recuento histórico, funcionaba el Instituto Materno Infantil, creado por el Acuerdo 14 de 1944,

Profesor Titular, Emérito y Honorario de la Universidad Nacional. Miembro Fundador de la Sociedad Colombiana de Historia de la Medicina. emanado de la Junta General de la Beneficencia de Cundinamarca. Hasta entonces el servicio de Maternidad del Hospital San Juan de Dios había tenido que soportar solo la creciente demanda de atención obstétrica venida de una población igualmente creciente. Comenzaba ya a hacer su aparición la explosión demográfica, que obligó a crear un gran hospital: el Instituto Materno Infantil. Se le dio el nombre de "Concepción Villaveces de Acosta" en homenaje a la progenitora del profesor José del Carmen Acosta Villaveces, director del Instituto, que en sus manos llegó a convertirse en el epicentro de la obstetricia y la neonatología nacionales. Muchos y muy importantes médicos se hicieron especialistas allí, para dispersarse por toda la geografía patria.

Hay otro hito histórico que no puede preterirse: La fundación de la Sociedad Colombiana de Obstetricia el 30 de marzo de 1943 en el Servicio de Maternidad del Hospital San Juan de Dios, por iniciativa del doctor Rafael Peralta Cayón. Sin duda, este hecho contribuyó grandemente al desarrollo de las disciplinas obstétricas, y sirvió de puente para la integración con la ginecología, pues el 21 de mayo de 1947 se convirtió en la Sociedad Colombiana de Obstetricia y Ginecología. En Bogotá, los ginecólogos estaban entonces liderados por Arturo Aparicio Jaramillo, y en Medellín por Pedro Nel Cardona.

El profesor Aparicio Jaramillo era docente de la Universidad Nacional, pero sus cuarteles estaban en el Hospital San José, donde nació la Sociedad de Cirugía de Bogotá. Precisamente, fue ésta la que auspició la Primera Convención Nacional de Ginecología, llevada a cabo en el mismo Hospital San José del 23 al 29 de mayo de 1949. En ella participó lo más granado de la ginecología nacional: Arturo Aparicio, por supuesto, Guillermo López Escobar, Hernando Amaya León, Jorge Amorocho Carreño, Antonio Ucrós Cuéllar, Gustavo Isaza Mejía, René Díaz Correa, Omar Campo M., Manuel Aristizábal, Cecilia Espinosa de Delgadillo, Alberto Villaneda Soto, Alvaro Espinosa, entre otros.

Asociados para actividades gremiales y científicas, los obstetras y los ginecólogos vieron la necesidad de contar con un vehículo de expresión, que diera cabida a sus inquietudes.

Ya antes, en 1945, las directivas de la recién creada Sociedad Colombiana de Obstetricia promovieron la publicación de un Boletín Científico, que se transformó en 1950 en la Revista Colombiana de Obstetricia y Ginecología.

Como vemos, estaban dadas las condiciones para que se realizara un primer encuentro de los obstetras y los ginecólogos de todo el país. Pero a las anteriores se sumó otra de particular importancia: comenzaba a adquirirse conciencia acerca de la necesidad y conveniencia que los 
médicos hicieran un frente común. La creación del Seguro Social la había despertado, había sido el toque a rebato, tal como lo dijera Arturo Aparicio Jaramillo en el discurso inaugural: "Una campanada de alerta, oída a través del Instituto de Seguro Social, vino a aglutinar a los médicos en derredor de sus respectivas sociedades, en el ánimo de luchar por sus fueros colectivos de dignidad, justicia y trabajo, que el Seguro quería menospreciar".

\section{La primera Convención}

En 1953 las directivas de la Sociedad Colombiana de Obstetricia y Ginecología estaban conformadas por Arturo Aparicio Jaramillo, que la presidía; por Carlos Roberto Silva Mojica en calidad de vicepresidente; Fernando Tamayo O., como secretario general; Hernando Navas Angel, secretario de actas, y Santiago Lleras Codazzi, tesorero. A ellos correspondió organizar uno de los congresos médicos más concurridos de cuantos se habían realizado antes en nuestro país. Se escogió para tal efecto el Gran Salón del Hotel Tequendama, recientemente inaugurado.

Aquella magna reunión recibió el nombre de Convención que, a decir verdad, no era el más apropiado para el efecto.

En cambio, con acertada justicia se designó presidente de la misma al profesor José del Carmen Acosta Villaveces, quien a la sazón era decano de la Facultad de Medicina de la Universidad Javeriana, presidente de la Federación Médica Colombiana, presidente de la Confederación Médica Panamericana y director científico del Hospital San Juan de Dios de Bogotá. El doctor Acosta acumulaba, pues, suficientes méritos, más que cualquier otro obstetra o ginecólogo. Era considerado como el gran maestro de la obstetricia.

La vicepresidencia estuvo a cargo de Rodulfo Camero Castaño, ginecólogo de prestigio en Bogotá, como que dirigía una clínica privada de su propiedad, una de las primeras, junto con la Clínica Calvo, destinada exclusivamente a la atención de partos y cirugía ginecológica. A los doctores Acosta y Camero los acompañaron Miguel Alvaro Fernández Bastidas, desde la secretaría general, Alvaro Espinosa y Espinosa como secretario de actas, y Santiago Lleras, como tesorero.

Debe establecerse que la Convención se llevó a cabo en momentos de paz y de euforia nacionales, pues todavía se vivía la luna de miel que el país disfrutó con el advenimiento al poder del general Gustavo Rojas Pinilla, complacencia que por cierto no duró mucho. La instalación se cumplió el 9 de septiembre y, como era lógico, estuvo a cargo del ministro de Salud Pública, Bernardo Henao Mejía. Los organizadores ofrecieron un coctel de inauguración en el Hotel Tequendama, en honor de las altas autoridades, civiles y militares. Un coronel del ejército, Julio Cervantes, era el Alcalde Mayor de la ciudad.

Un total de 224 médicos, venidos de diferentes partes del país, quedaron registrados como asistentes a la Convención. No todos eran obstetras o ginecólogos de escuela. La mayor parte la constituían médicos generales, que, de seguro, ejercían ambas especialidades. Como se anotó atrás, la obstetricia y la ginecología se hallaban divorcia- das, se mantenían en estado de pureza académica, vale decir que cada cual estaba confinada a su propio campo de acción, no obstante que las dos tuvieran que ver con los mismos órganos anatómicos.

Dado que se trataba de una reunión de carácter nacional, hubo representación oficial de casi todas las regiones. Veamos aquellas distintas a Bogotá y Cundinamarca: Antioquia: Pedro Nel Cardona, Armando Posada y Jorge Henao Posada.

Atlántico: Fabián Jiménez, José María Garrido Gómez y Eduardo Acosta Bendeck.

Bolívar: Napoleón Franco Pareja.

Boyacá: Alberto Espejo y Guillermo Zubieta.

Caldas: Eutimio Rubio y Bernardo Botero Peláez.

Córdoba: Ricardo Bechara.

Chocó: Julio Figueroa Villa.

Huila: César Beltrán y Luis Felipe Díaz Rivera.

Magdalena: José María Serna.

Nariño: Francisco Conto.

Norte de Santander: Alberto Duarte Contreras y Manuel J. Arenas.

San Andrés y Providencia: Adalberto Gallardo.

Santander: Primitivo Rey Rey, Fabio Durán Velasco e Isaías Arenas Buenahora.

Valle del Cauca: Tomás Becerra y Hugo Campos.

Además de médicos que ejercían en Bogotá una de las dos disciplinas, asistieron unos cuantos -por cierto muy distinguidos- dedicados a otras especialidades. Entre ellos figuraron los pediatras Ernesto Plata Rueda y Humberto Gómez Herrera; los urólogos Jorge Cavelier, Alfonso Ramírez Gutiérrez y Luis Alberto Blanco; el internista Luis Forero Nogués; los laboratoristas Hernando Gómez Vesga y César Mendoza; los cirujanos Raúl Bernet y José Tomás Henao; el radiólogo Gonzalo Esguerra Gómez.

Como ya dijimos, el discurso de inauguración estuvo a cargo del presidente de la Sociedad de Obstetricia y Ginecología, Arturo Aparicio Jaramillo. Su contenido permite reafirmar lo que atrás registramos: las dificultades que atravesaba el cuerpo médico a causa de la creación y funcionamiento de la seguridad social; su acercamiento en el seno de la Sociedad con fines gremiales y científicos; la separación de la obstetricia y la ginecología en la cátedra y en el ejercicio. No podía faltar en esta ocasión inaugural una referencia a algo de especial importancia cuando se revisa la evolución de la ginecología entre nosotros: la introducción del concepto funcional frente al concepto anatómico, que había primado hasta cuando el mismo Arturo Aparicio en Bogotá y Pedro Nel Cardona en Medellín, hicieron ver que el manejo de la paciente ginecológica no era solo del dominio de la cirugía.

Al tiempo que rendía un homenaje a los pioneros de las dos especialidades, Aparicio incitó a sus practicantes de entonces a colocarse a tono con los progresos alcanzados en otras latitudes. "A la par que la ciencia avanza -decía-, necesitamos permanente renovación; necesitamos indagar, estudiar, promover frecuentes reuniones de esta índole, ver otros horizontes para cambiar un poco nuestra mentalidad y no encasillarnos en nuestro propio y egoísta saber".

Para hacer consistente ese criterio y para fortalecer a la Sociedad, fue aprobada la proposición siguiente, presentada por la mesa directiva: 
"Todos los obstetras y ginecólogos asistentes a la Primera Convención Nacional de Obstetricia y Ginecología, que hasta el momento no pertenezcan a la Sociedad Colombiana de Obstetricia y Ginecología, serán considerados en adelante como Miembros Correspondientes de ella.

Los Miembros Afiliados de la Sociedad que hayan presentado trabajos en la Convención, pasarán de hecho a ser Miembros de Número.

Los internos de las especialidades obstétrica y ginecológica que hayan colaborado en los trabajos presentados, serán de hecho Miembros Afiliados".

En este encuentro histórico, la revisión y análisis de las conclusiones oficiales de la Convención permiten deducir cuáles fueron los temas que ocuparon la atención de los participantes, como también conocer el estado de los mismos. Por eso es de necesidad transcribir íntegro el informe de conclusiones, elaborado por una comisión ad hoc compuesta por los doctores Rafael Peralta Cayón, Eduardo Acosta Bendeck, Bernardo Botero Peláez, Tomás Becerra, Pedro Nel Cardona, Primitivo Rey Rey y Hernando Amaya León. Dice así:

\section{Temas obstétricos}

1. Toxicosis gravídicas. Todavía es delicada la toxicosis gravídica por razones etiológicas y por los índices de mortalidad materna y fetal, al mismo tiempo que persiste el desacuerdo terapéutico, ya que hay controversia entre los tratamientos médico puro y obstétrico puro. En tal sentido, las conductas deben tener la mira de salvar las dos vidas hasta donde sea posible, y debe intensificarse el tratamiento profiláctico en consultas prenatales bien organizadas.

2. Factor Rh. La convención considera: Que es imprescindible la determinación del Factor Rh siempre que se vaya a administrar sangre a una mujer a fin de asegurar que ésta sea compatible para evitar los graves inconvenientes de la inmunización anti-Rh.

Que es indispensable la determinación del Factor Rh en toda gestante, así como el control de aglutininas anti-Rh en las gestantes Rh negativas con esposo Rh positivo, a fin de estar prevenidos para adoptar una conducta apropiada.

Que es indispensable el control clínico y hematológico de todo niño hijo de madre Rh negativa, aun en ausencia de isoinmunización demostrable en ésta.

Que aunque hasta el presente se ha laborado con la conducta consistente en la inducción del parto prematuro, la opinión, así extranjera como nacional, más aceptada hoy, es la de procurar los partos a término de las gestantes Rh negativas.

Se desaconseja completamente la vía del seno longitudinal superior para practicar transfusiones a los recién nacidos.

3. Temas libres. Merecieron interés y es recomendable seguir su estudio, los siguientes: a) Nueva técnica de embriotomía con el parto de Turpin; b) Los procedimientos de pelvimetría en radiodiagnóstico obstétrico y el interés de su divulgación;

c) La maniobra de Ramírez Merchán para la aplicación de fórceps en variedades transversas y posteriores de vértice, d) la mortalidad materna en clínicas obstétricas: es todavía alarmante el índice hospitalario de Bogotá, como es lujoso el de clínicas diferentes y no es escasa la incidencia de las toxicosis. Si en el primero, además del estado precario del elemento paciente, hay factores de organización interna determinantes, sería necesario revisarlos; e) el concepto del encajamiento, tendiente a demostrar que es precoz en la primigestante como excepción pero no como regla.

\section{Temas ginecológicos}

1. Endometriosis. Apenas se esbozó este tema, a pesar de haber sido oficialmente señalado, en dos trabajos, y necesita nuevos estudios especialmente desde el punto de vista histopatológico, por lo cual podría recomendarse como tema para futuras convenciones.

2. Esterilidad. Es un problema de trascendencia en ginecología y de novedad entre nosotros. Apenas se inicia la consulta de esterilidad en Bogotá, Medellín y Bucaramanga y es de desear que los demás centros ginecológicos sigan el mismo derrotero. Debe imponerse como condición para iniciar el estudio de la pareja estéril el examen primero del marido a fin de evitar inútiles erogaciones en tiempo y dinero, por la resistencia del varón."

Como vemos, hace 43 años, que fue cuando tuvo cumplimiento la Convención que comentamos, los dos temas oficiales de obstetricia fueron la toxemia gravídica y la isoinmunización por Factor Rh. La primera era la principal causante de las muertes maternas y fetales. El doctor Carlos Roberto Silva presentó un estudio comparativo de mortalidad materna, muy interesante, tomando como material los decesos ocurridos en clientela privadá, en pacientes del Instituto de Seguros Sociales y en aquellas hospitalarias. Sus resultados eran de esperar: la toxemia gravídica (preeclampsia y eclampsia) ocupó el primer lugar en los tres grupos estudiados, no obstante que los índices de mortalidad general fueron diferentes: de $0.58 \%$ en el de clientela particular, de $1.68 \%$ en el del Seguro Social, y de $8.4 \%$ en el de pacientes atendidas en el Instituto Materno Infantil, donde la sola eclampsia dio cuenta de $19 \%$ de las muertes maternas, hecho éste que le permitió aconsejar al doctor Silva que se tuviera presente "que la insistencia en un tratamiento, cuando la clínica y el laboratorio indican agravamiento de la eclampsia, conduce al riesgo de perder dos vidas; en cambio, el acudir oportunamente a la vía quirúrgica asegura por lo menos una vida". Por su parte, el profesor Luis María Ferro, refiriéndose a la prevención de la enfermedad decía: "Las toxicosis gravídicas constituyen el más serio capítulo de la patología obstétrica. Su tremenda gravedad, la rapidez de su evolución, la morbilidad y la mortalidad elevadísimas, justifican toda la lucha tenaz para su profilaxis y precoz diagnóstico. Es nuestra opinión sincera, apoyada en la propia experiencia, que la vigilancia estricta del embarazo en las consultas prenatales es la mejor arma que poseemos para combatir tan terribles complicaciones..." El doctor Acosta Villaveces, quien dirigió hasta poco antes de la Convención el Instituto Materno Infantil, llevó a cabo una revisión de los tratamientos para la toxemia gravídica utilizados allí, que le permitió manifestar que "como para toda terapéutica, la base de la indicación es el estudio clínico minucioso del caso para saber de qué forma de la toxicosis se trata y en qué condiciones se halla". Para entonces se 
hablaba de las formas "seca" y "húmeda" de la toxemia gravídica, según la presencia o no de edemas. De ahí que se recomendara un balance estricto de los líquidos suministrados y eliminados. Una forma de estimular la excreción en las pacientes edematizadas era a través de la mucosa intestinal, administrando solución saturada de sulfato de magnesio, en cucharadas repetidas hasta producir una verdadera diarrea serosa, o "sangría blanca". "Esta derivación del agua -afirmaba el doctor Acostacontenida en el aparato vascular disminuye la plétora sanguínea y por consiguiente baja la tensión arterial, descenso que es coadyuvado por la acción sedante del magnesio". A más de lo anterior, la movilización de líquidos se propiciaba con la administración intravenosa de solución glucosada al $50 \%$ en cantidad de 40 a $50 \mathrm{cc}$, cada 4 a 6 horas. En las llamadas "preeclampsias graves", o "inminencias de eclampsia", se aplicaban barbitúricos en dosis de 20 a 25 centigramos, por vía intramuscular.

En las formas "secas" el plan terapéutico consistía en inyección intramuscular de extracto fluido de veratrum viride, en dosis de $0.6 \mathrm{cc}$, asociado a la solución de sulfato de magnesio, también intramuscular, y a la inyección intravenosa de 1.000 a $1.500 \mathrm{cc}$ de dextrosa al $10 \%$. Aparecidas las convulsiones, vale decir la eclampsia, a pesar del tratamiento anterior, se recurría a la punción lumbar para extraer 30 a $40 \mathrm{cc}$ de líquido cefalorraquídeo, "para disminuir la tensión intracraneana y la irritación de la corteza cerebral determinante de las convulsiones".

En cuanto a la isoinmunización por factor $\mathrm{Rh}$, o eritroblastosis fetal, el encargado de hablar del asunto fue el doctor Ernesto Plata Rueda, que era el jefe de las sala-cunas del Instituto Materno-Infantil y de la Clínica David Restrepo. En realidad, no se trataba de un problema que justificara su inclusión en la agenda de la Convención como tema prioritario. El mismo doctor Plata señalaba en su exposición que la frecuencia era realmente escasa, como que la incidencia de madres $\mathrm{Rh}$ negativas era de $6.3 \%$, a diferencia de la reportada en otras latitudes de población blanca; $15 \%$. De novedoso, el ponente, que comenzaba a sobresalir en el escenario de la pediatría nacional, habló de la sustancia Hapteno Rh, no disponible en Colombia, "extraida de glóbulos rojos y carente de poder antigénico, que se apoderaría de los anticuerpos anti $\mathrm{Rh}$ in vivo". Sin duda, no se trataba propiamente de la gammaglobulina anti Rh, pues fue en 1964 cuando Vicente Frida la ensayó por vez primera en humanos, en la cárcel de Sing-Sing. Mencionó también el doctor Plata el empleo de vitamina $\mathrm{E}$ y hormona del cuerpo lúteo, que "favorecen la implantación y la integridad placentaria. Ambas son inocuas y se justifica ahora su uso".

Correspondió al profesor Pedro Nel Cardona, de la Facultad de Medicina de la Universidad de Antioquia, encargarse del tema "endometriosis", entidad que sólo llevaba tres décadas de conocida y, por lo tanto, de particular interés para los ginecólogos. El doctor Cardona presentó su experiencia, basándose en 27 casos estudiados en un lapso casi de siete años, y cuyo tratamiento fue quirúrgico en todos: 22 histerectomías y el resto extirpaciones focales. Un anuncio importante fue el tratamiento médico: la gran novedad consistía en "la estrogenoterapia sostenida por un lapso de 3 ó 4 meses, con dosis de 5 miligramos cada 4 días de etilbestrol, y en el momento de la menstruación 5 miligramos cada 15 minutos hasta la supresión de la hemorragia, que obra inhibiendo la secreción de las hormonas hipofisiarias, con lo cual parece que las lesiones endometriales entran en franca regresión".

A la "esterilidad matrimonial" se le dio gran beligerancia, pues se prestó para un prolongado simposio a cargo del Departamento de Ginecología del Hospital San José, cuyo jefe de la sección de Esterilidad era el profesor Aparicio Jaramillo. Los distintos factores involucrados en el problema fueron tratados por distintos expositores, la mayoría de ellos ginecólogos. Intervinieron también endocrinólogos, laboratoristas, patólogos, radiólogos y urólogos.

Los redactores de las conclusiones llamaron la atención acerca de algunos de los temas libres, todos de carácter obstétrico. Recuérdese que para aquella época la culminación del embarazo por vía vaginal era el triunfo de la obstetricia y la gran satisfacción del tocólogo. El doctor Rafael Peralta se esmeraba por demostrar que el encajamiento temprano de la presentación cefálica, en pacientes primigestantes, no era tan frecuente como lo pregonaban escuelas foráneas. Rafael Ramírez Merchán, por su parte, continuaba divulgando y defendiendo la maniobra instrumental, conocida con su nombre, encaminada a rotar con una de las cucharas del fórceps la cabeza fetal que, por presentarse en una variedad de vértice posterior o transversa, hacía difícil el parto vaginal. Como él, muchos de sus discípulos llegaron a dominar la técnica, gracias a lo cual los nacimientos atendidos por ellos terminaban felizmente. El profesor de radiología, Gonzalo Esguerra Gómez, y Alberto Cárdenas Escobar, director de la Clínica David Restrepo, dieron a conocer un trabajo -el primero en nuestro medio- sobre el empleo de la radiopelvimetría en la identificación de las desproporciones cefalopélvicas, es decir, encaminado a sentar un pronóstico que diera plena justificación a la práctica de la operación cesárea. No obstante los distintos métodos utilizados (isométricos, matemáticos, estereoscópicos), la radiopelvimetría no llegó nunca a ocupar un lugar de importancia en la práctica obstétrica. Finalmente, el doctor Gustavo Riaño Alvarez, entonces Jefe de Clínica en el Instituto Materno Infantil, dio a conocer en la Convención el llamado "embriótomo de Torpin", que tenía como finalidad decapitar el feto muerto cuyo parto vaginal era físicamente imposible a causa de haber buscado salida presentando uno de sus hombros. El mérito de la comunicación del doctor Riaño radicó en haber modificado la técnica del doctor Torpin -que era director del Medical College of Georgia-, pues en vez de decapitación pura propuso la sección o corte "en banda presidencial”, más segura, fácil y rápida.

Entre las conclusiones varias de la Primera Convención Colombiana de Obstetricia y Ginecología se acordó que la siguiente reunión debería cumplirse en la ciudad de Cali, pasado dos años, aprovechando que para entonces el Colegio Nacional de Cirujanos tendría allí su tercera convención. Los temas aprobados oficialmente fueron: "Cáncer del cuello uterino", "Shock obstétrico" y, nuevamente, "Factor Rh". De esa manera quedaban institucionalizados los congresos de ginecobstetricia, de tanta importancia en el desarrollo de ésta disciplina. 